\title{
La educación post-Covid y el alumnado con discapacidad $o$ con necesidades específicas de apoyo educativo: marco normativo y experiencias ${ }^{\text {*.: }}$
}

\section{Post-Covid education and students with disabilities or with specific educational support needs: normative framework and experiences ${ }^{*}$}

\author{
Lluc Massaguer Busqueta \\ Profa. Estudios de Ciencias de la Información y de la Comunicación \\ Universitat Oberta de Catalunya, España \\ E-mail: $\underline{\text { lmassaguerb@uoc.edu }}$
}

\section{Rubén Alcaraz Martínez}

Prof. asociado de la Facultad de Información y Medios Audiovisuales

Universidad de Barcelona

E-mail: ralcaraz@ub.edu

\section{Mireia Ribera Turró}

Profa. agregada del Departamento de Matemáticas e Informática

Universidad de Barcelona

E-mail: ribera@ub.edu

\footnotetext{
* El Servicio de Apoyo a las Personas con Discapacidad en la Universidad (SAPDU) usa este término: estudiantes con discapacidad y/o con Necesidad Específica de Apoyo Educativo (en adelante NEAE), para equipararlo a la nomenclatura de la educación no universitaria.

* Esta investigación ha sido financiada por la Convocatoria de Ayudas a la Investigación en Docencia Universitaria del Instituto de Desarrollo Profesional de la Universidad de Barcelona, REDICE-20

* The Support Service for People with Disabilities at the University (SAPDU) uses this term: students with disabilities and / or with a Specific Need for Educational Support (hereinafter NEAE), to equate it with the nomenclature of non-university education.
} 


\title{
Marina Salse Rovira
}

Profa. titular de la Facultad de Información y Medios Audiovisuales

Universidad de Barcelona

E-mail: salse@ub.edu

\section{Rosa M. Satorras Fioretti}

Profa. Titular de Derecho Eclesiástico del Estado

Facultad de Derecho

Universidad de Barcelona

E-mail: satorras@ub.edu

\begin{abstract}
Resumen: El objetivo de esta investigación es presentar un análisis de las reflexiones, valoraciones y peticiones del profesorado, alumnado y personal de gestión universitario en relación a la docencia para alumnado con necesidades específicas de aprendizaje, en un marco legal explícito de obligaciones y compromisos adquiridos. El estudio se enmarca en la experiencia de la docencia online o híbrida durante la pandemia de la COVID-19 y se comparan las declaraciones de las personas implicadas con las medidas publicadas por las universidades y las buenas prácticas existentes. Las cuestiones a las que se pretende dar respuesta son: ¿Qué medidas son importantes para el estudiantado con necesidades específicas en situaciones como la vivida? ¿Cuáles lo son para el profesorado? ¿Cómo podemos prepararnos ante nuevas situaciones de emergencia? La metodología de investigación ha consistido en una revisión documental del marco normativo y legislativo aplicable, así como en el análisis temático con base en las transcripciones de 45 entrevistas semi-estructuradas realizadas a estudiantes (27), docentes (10), y personal con responsabilidades relacionadas con el estudiantado con necesidades específicas del aprendizaje (8). Las cuestiones más problemáticas detectadas tanto por estudiantes como por docentes y personal de gestión son la no accesibilidad total de las clases en línea, las aulas virtuales, los materiales docentes, así como de los exámenes o pruebas de evaluación. Por parte del estudiantado, se destaca también el escaso acompañamiento emocional/social con el que han contado.
\end{abstract}

Palabras clave: Docencia, Enseñanza superior, Accesibilidad digital, Competencias digitales, Covid-19. 


\begin{abstract}
The aim of this research is to summarise thoughts, feelings and demands of lecturers, students and support staff in relation to special needs education, within the context of a legal framework with stated compromises and legal duties. The research is contextualized in the COVID-19 pandemics online or hybrid teaching, and it compares the statements of stakeholders with the recommendations issued by universities during this period and with existing best practices. The article aims to give an answer to the following questions: Which are the most important measures for students with special needs in this situation? Which are the most important for lecturers? How can we get ready for similar emergency situations? The methodology consisted in a review of the legal and standard framework, and a thematic analysis of 45 semi-structured interviews to students (27), lecturers (10), and support staff (8). The most problematic areas for students, lecturers and support staff are the inaccessibility of online teaching, virtual classrooms, teaching material, exams and assessment exercises. Students also emphasize the need for emotional/social support and the lack of $i t$.
\end{abstract}

Keywords: Teaching, Higher education, Digital accessibility, Digital skills, Covid-19.

\title{
1. Introducción
}

\subsection{Motivación y contexto}

Tras el decreto del 14 de marzo de 2020 por el que se declara el estado de alarma para la gestión de la crisis sanitaria ocasionada por el COVID-19, se suspende toda actividad presencial en la enseñanza universitaria, recogiéndose, asimismo, la necesidad de mantener tales actividades educativas en modalidades a distancia y en línea. 
Sin duda, uno de los principales desafíos a los que han tenido que dar respuesta las universidades ha sido la gestión de este cambio abrupto y de emergencia desde el modelo de enseñanza presencial a uno virtual en un margen de tiempo insuficiente. Esto ha comportado la adaptación de toda una serie de cuestiones relacionadas con el proceso de enseñanza-aprendizaje que debían adaptarse no sólo al contexto virtual, sino también al contexto particular derivado de la crisis sanitaria. Si bien algunas universidades cuentan con cierta experiencia en la creación, planificación y gestión de programas formativos en línea, la mayor parte de su profesorado nunca se ha enfrentado a ello y ha tenido que adaptar, en tiempo récord, la metodología y el plan docente a la nueva situación.

Esta situación ha representado un desafío importante para todo el colectivo universitario y ha tenido un impacto negativo en la satisfacción, motivación, eficacia y compromiso del estudiantado (AGUILERA-HERMIDA, 2020), el cual ha vivido, además, la confusión y sensación de inseguridad derivada de un menor contacto con el personal académico y de soporte (HILL y FITZGERALD, 2020, 4-6), así como con el resto de estudiantes.

En particular, uno de los grupos más vulnerables, el alumnado con necesidades específicas de apoyo educativo, ha vivido un reto aún mayor. La situación de emergencia ha provocado, entre otros, el olvido de pautas de adaptación ya asentadas en las universidades que facilitaban el proceso de enseñanza-aprendizaje, o el uso de nuevas plataformas tecnológicas que no respondían a sus necesidades.

\subsubsection{Marco legal}

La accesibilidad de los materiales, las clases y los entornos en la Universidad son una obligación legal establecida en diversos instrumentos normativos aplicables tanto en España como en sus autonomías. Algunas universidades también han publicado pautas para concretar estos requisitos. En este estudio se mencionan las publicadas por la Universidad de Barcelona por ser una de las mayores, con más disciplinas y más estudiantes de España y, en consecuencia, se estudian las medidas tomadas también por el gobierno de Cataluña. 
En el ámbito supranacional, la Convención internacional sobre los derechos de las personas con discapacidad, aprobada por la Asamblea General de Naciones Unidas el 13 de diciembre de 2006 y ratificada por España en 2007 establece en su art. 24 que «Los Estados Partes asegurarán que las personas con discapacidad tengan acceso general a la educación superior, la formación profesional, la educación para adultos y el aprendizaje durante toda la vida sin discriminación y en igualdad de condiciones con las demás. A tal fin, los Estados Partes asegurarán que se realicen ajustes razonables para las personas con discapacidad».

Asimismo, la Resolución del Consejo de Europa. Res AP (2007) 3 para “Alcanzar la plena participación a través del Diseño Universal" recomienda a todos los gobiernos de la UE a iniciar acciones para incorporar en todos los procesos de la sociedad, incluido el educativo, del llamado Diseño Universal del Aprendizaje (DUA), que supone la creación desde el origen de entornos, productos, tecnologías y servicios de información accesibles para todas las personas sin necesidad de adaptación o de soluciones especiales; es decir, que no se trata ya de adaptarse a posteriori a las necesidades de las personas con capacidades diferentes, sino de diseñarlo todo a priori para que todas las personas puedan acceder sin discriminación.

De la Convención internacional se hizo eco en España el Real Decreto Legislativo 1/2013, de 29 de noviembre, por el que se aprueba el Texto Refundido de la Ley General de derechos de las personas con discapacidad y de su inclusión social, que fija en su art. 18.2 que «Corresponde a las administraciones educativas asegurar un sistema educativo inclusivo en todos los niveles educativos así como la enseñanza a lo largo de la vida (...), prestando atención a la diversidad de necesidades educativas del alumnado con discapacidad, mediante la regulación de apoyos y ajustes razonables para la atención de quienes precisen una atención especial de aprendizaje o de inclusión». A mayor abundamiento, en su art. 20.c), referido a los estudios universitarios, exige que las pruebas de evaluación también se deben adaptar a las características de la discapacidad que presente el alumnado; y, además, establece el sistema sancionador en el caso de que sea una entidad pública la que conculque estos derechos, considerando como infracción administrativa grave (arts. 80 y 81) el incumplimiento de las exigencias de accesibilidad y de realización de ajustes razonables. 
Entrando en la más concreta Ley Orgánica 6/2001, de 21 de diciembre, de Universidades, la D.A. $24^{\text {a }}$, referida toda ella a la inclusión de las personas con discapacidad, preceptúa la implementación de medidas de acción positiva que eviten la discriminación directa o indirecta del alumnado con discapacidad en todos los ámbitos, así como la promoción de acciones para que todos ellos dispongan de los medios, apoyos y recursos que aseguren su igualdad real y efectiva. Y, en concreto, «los edificios, instalaciones y dependencias de las universidades, incluidos también los espacios virtuales, así como los servicios, procedimientos y el suministro de información, deberán ser accesibles para todas las personas, de forma que no se impida a ningún miembro de la comunidad universitaria, por razón de discapacidad, el ejercicio de su derecho a ingresar, desplazarse, permanecer, comunicarse, obtener información u otros de análoga significación en condiciones reales y efectivas de igualdad». Asimismo, el Real Decreto 1791/2010, de 30 de diciembre, por el que se aprueba el Estatuto del Estudiante Universitario, establece en su art. 65 la exigencia a las universidades españolas de garantizar la accesibilidad de las herramientas, de los formatos y de las páginas web, facilitando en todo caso la posibilidad de descargar la información.

Por su parte, la Ley catalana 13/2014, de 30 de octubre de accesibilidad, algo más cicatera que la española y la internacional, exige de forma general a todos los proveedores de servicios públicos la adopción de «las mejores condiciones de accesibilidad posibles» (art. 26) y, en especial en el ámbito educativo, la garantía de accesibilidad al alumnado «con discapacidad sensorial, ya sea auditiva, ya sea visual, ya sea auditiva y visual a la vez, y también a los alumnos con dificultades graves de lectura o de comprensión» (art. 32). Obviamente, la normativa española e internacional son directamente aplicables en Cataluña, por lo que todos estos términos lo son de forma acumulativa.

Para terminar, en el marco específico de la Universidad de Barcelona, encontramos en sus Estatutos (bastante antiguos, de 2003) la garantía de promover la integración de las personas con discapacidad (art. 4.2), adecuando sus instalaciones y el fomento del acceso y la integración de las mismas (art. 121.2). De todo lo anterior se deduce la clara obligación actual que tienen las Universidades españolas de hacer accesibles a todas las personas los entornos, las clases, los materiales, los recursos y las instalaciones. 
1.1.1.1 Traslación del marco legal a las regulaciones universitarias surgidas en el contexto de la pandemia

La situación de pandemia generó la necesidad de regular con más concreción la actividad académica durante el periodo de emergencia. En un estudio previo (MASSAGUER et al., 2021) se recogieron las disposiciones publicadas por universidades españolas públicas que hacían mención al alumnado con necesidades especiales. Se complementó el análisis con la inclusión de diversas guías de buenas prácticas para la creación de documentos accesibles, que aparecían citados en dichas resoluciones. En estas disposiciones, y en las guías de referencia mencionadas en ellos, sí se recogían bastantes concreciones de buenas prácticas para atender al alumnado con necesidades especiales.

\subsubsection{Traslación del marco legal en las competencias digitales de los docentes}

La implementación de las medidas de accesibilidad depende, en gran medida, del cuerpo docente y de sus competencias. En concreto, cuando hablamos de enseñanza virtual, toman una gran importancia sus competencias digitales. Por ello, en este apartado analizamos si los requisitos legales referentes a la educación inclusiva se han recogido en los dos marcos de competencias digitales de referencia en España, DigCompEdu e INTEF.

El Marco europeo para la competencia digital de los educadores (DigCompEdu) (PUNIE y REDECKER, 2017) reconoce un total de 22 competencias en 6 áreas diferentes: 1) compromiso profesional (uso de las tecnologías digitales para la comunicación, la colaboración y el desarrollo profesional); 2) contenidos digitales (búsqueda, creación e intercambio de contenidos digitales); 3) enseñanza y aprendizaje (gestión y organización del uso de las tecnologías digitales en la enseñanza y el aprendizaje); 4) evaluación y retroalimentación (utilización de tecnologías y estrategias digitales para mejorar la evaluación); 5) empoderamiento de los estudiantes (uso de las tecnologías digitales para mejorar la inclusión, la personalización y el compromiso activo del alumnado con su propio aprendizaje), y 6) desarrollo de la competencia digital de los estudiantes (capacitación de los estudiantes para utilizar de forma creativa y responsable las tecnologías digitales para la información, la comunicación, la creación de contenidos, el bienestar y la resolución de problemas). Si bien DigCompEdu supone un buen punto de 
referencia para la formación tecnológica, no hace especial foco en el alumnado con necesidades específicas ni se concretan los requisitos que éstos necesitan a nivel de formación tecnológica.

Por su parte, en España, el Marco común de competencia digital docente nace en 2012 como parte del Plan de cultura digital en la escuela (INTEF, 2012), y del Marco estratégico de desarrollo profesional docente (INTEF, 2012), con el objetivo de ser una referencia útil con fines de formación, así como en procesos de evaluación y acreditación docente (INTEF, 2017). La lista de competencias identificadas resulta especialmente valiosa en un contexto en el que, según recoge el mismo Plan de cultura digital en la escuela, la Universidad "no forma suficientemente al futuro profesor en competencia digital [...ni tampoco supone...] un requisito para el ejercicio de la docencia”. El Marco común..., adaptado a partir del DigCompEdu, reconoce cinco grandes áreas: 1) información y alfabetización informacional (identificar, localizar, obtener, almacenar, organizar y analizar información digital, datos y contenidos digitales, evaluando su finalidad y relevancia para las tareas docentes); 2) comunicación y colaboración (comunicar en entornos digitales, compartir recursos a través de herramientas en línea, conectar y colaborar con otros a través de herramientas digitales, interactuar y participar en comunidades y redes; conciencia intercultural); 3) creación de contenidos digitales (crear y editar contenidos digitales nuevos, integrar y reelaborar conocimientos y contenidos previos, realizar producciones artísticas, contenidos multimedia y programación informática, saber aplicar los derechos de propiedad intelectual y las licencias de uso); 4) seguridad (protección de información y datos personales, protección de la identidad digital, protección de los contenidos digitales, medidas de seguridad y uso responsable y seguro de la tecnología); y 5) resolución de problemas (identificar necesidades de uso de recursos digitales, tomar decisiones informadas sobre las herramientas digitales más apropiadas según el propósito o la necesidad, resolver problemas conceptuales a través de medios digitales, usar las tecnologías de forma creativa, resolver problemas técnicos, actualizar su propia competencia y la de otros). Para cada una de estas áreas se identifican un total de 21 competencias y se establecen seis niveles competenciales: básico (A1 y A2), intermedio (B1 y B2) y avanzado (C1 y C2). De forma similar al caso anterior, este marco tampoco hace ningún énfasis, ni específica, la formación para las necesidades de educación especial. 
En conclusión, se puede observar que no ha habido una traslación explícita de las obligaciones legales respecto a la educación inclusiva en estos marcos de referencia y que los cuerpos docentes desconocen sus obligaciones, las necesidades y los recursos tecnológicos para atender con calidad al perfil del alumnado con necesidades específicas de aprendizaje.

\subsubsection{Colección de buenas prácticas}

A partir de las recomendaciones de las universidades y de las guías de creación de documentos accesibles, en un estudio previo (MASSAGUER et al, 2021), se sistematizaron y se agruparon las medidas bajo diferentes categorías temáticas, por medio de la técnica de diagramas de afinidad. Se identificaron un total de 99 medidas, clasificadas en 10 categorías: planificación (8 medidas), desarrollo de las clases en línea (8 medidas), tutorías (4 medidas), evaluación (29 medidas), metodologías de enseñanzaaprendizaje (8 medidas), materiales accesibles (20 medidas), acompañamiento emocional/social (3 medidas), logística/aplicación de la adaptación (11 medidas), aspectos legales ( 5 medidas) y servicios de atención ( 2 medidas).

El resultado de este análisis se puede consultar en el documento Informe de buenas prácticas metodología docente (ES)

\subsection{Objetivos}

El objetivo de esta investigación es presentar un análisis de las reflexiones, valoraciones y peticiones del profesorado, alumnado y personal de gestión universitario en relación con la docencia para el alumnado con necesidades específicas de apoyo educativo, en un marco legal explícito de obligaciones y compromisos adquiridos. El estudio se enmarca en la experiencia de la docencia en línea o híbrida durante la pandemia del COVID-19 y se comparan las declaraciones de los distintos sectores participantes con las medidas publicadas por las universidades y las buenas prácticas existentes, para analizar si éstas reflejan realmente las necesidades y peticiones de los implicados en el proceso de enseñanza-aprendizaje. Las cuestiones a las que se pretende dar respuesta son: ¿Qué medidas son importantes para el alumnado con necesidades específicas en situaciones 
como la vivida? ¿Cuáles lo son para el profesorado? ¿Cómo podemos prepararnos ante nuevas situaciones de emergencia?

\section{Metodología}

En este estudio se entrevistó a un total de 27 estudiantes seleccionados a partir de su perfil de diversidad para obtener una visión amplia de sus experiencias durante la pandemia. También se entrevistó a 10 docentes que habían impartido docencia a esos estudiantes con necesidades específicas de aprendizaje durante el periodo mencionado y a 8 profesionales (entre personal administrativo y docente con responsabilidades de atención a este alumnado). La tabla 1 recoge las características más relevantes de las personas entrevistadas.

Tabla 1. Codificación de las personas entrevistadas

\begin{tabular}{|c|c|c|c|c|c|c|c|}
\hline Id & Código & Rol & $\begin{array}{c}\text { Perfil y categoría } \\
\text { UNE EN } 301549 \\
(\mathbf{2 0 0 8 )}\end{array}$ & Estudios & $\begin{array}{l}\text { Curso en } \\
2020-21\end{array}$ & Género & Edad \\
\hline 1 & AA1 & Estudiante & $\begin{array}{c}\text { Síndrome de Asperger } \\
\text { (UNE10) }\end{array}$ & Ingeniería & $1^{\circ}$ & Mujer & 18 \\
\hline 2 & AA2 & Estudiante & $\begin{array}{c}\text { Trastorno bipolar } \\
\text { (UNE10) }\end{array}$ & $\begin{array}{l}\text { Ciencias } \\
\text { políticas }\end{array}$ & $1^{\circ}$ & Hombre & 33 \\
\hline 3 & AA3 & Estudiante & $\begin{array}{c}\text { Malformación de } \\
\text { Arnold Chiari } \\
\text { (UNE10) }\end{array}$ & Veterinaria & $2^{\circ}$ & Hombre & 29 \\
\hline 4 & AA4 & Estudiante & $\begin{array}{c}\text { Discapacidad auditiva } \\
\text { (UNE04) }\end{array}$ & $\begin{array}{l}\text { Filosofía y } \\
\text { letras }\end{array}$ & $2^{\circ}$ & Mujer & 52 \\
\hline 5 & AA5 & Estudiante & $\begin{array}{c}\text { Discapacidad visual } \\
\text { (UNE01) }\end{array}$ & Psicología & $1^{\circ}$ & Hombre & 34 \\
\hline 6 & AA6 & Estudiante & $\begin{array}{l}\text { Trastorno depresivo } \\
\text { (UNE10) }\end{array}$ & Medicina & $2^{\circ}$ & Hombre & 45 \\
\hline 7 & AE1 & Estudiante & $\begin{array}{c}\text { Ictus cerebral y } \\
\text { dificultad motora y } \\
\text { cognitiva (UNE10) }\end{array}$ & $\begin{array}{l}\text { Economía y } \\
\text { Empresa }\end{array}$ & $3^{\circ}$ & Hombre & 21 \\
\hline 8 & AE2 & Estudiante & $\begin{array}{c}\text { Discapacidad visual } \\
\text { (UNE01) }\end{array}$ & Estadística & $2^{\circ}$ & Hombre & 23 \\
\hline 9 & AE3 & Estudiante & Hemiparesia (UNE07) & $\mathrm{ADE}$ & $4^{\circ}$ & Hombre & 23 \\
\hline 10 & AE4 & Estudiante & $\begin{array}{c}\text { Discapacidad visual } \\
\text { (UNE01) }\end{array}$ & Sociología & $3^{\circ}$ & Hombre & 21 \\
\hline 11 & AE5 & Estudiante & Enfermedad de Crohn & $\mathrm{ADE}$ & $3^{\circ}$ & Hombre & 24 \\
\hline 12 & AE6 & Estudiante & $\begin{array}{l}\text { Esclerosis múltiple } \\
\text { (UNE01, } 07 \text { y 10) }\end{array}$ & Economía & $3^{\circ}$ & Mujer & 23 \\
\hline 13 & AE7 & Estudiante & $\begin{array}{l}\text { Síndrome Morquio } \\
\text { (UNE07 y 08) }\end{array}$ & Economía & $3^{\circ}$ & Hombre & 23 \\
\hline 14 & AE8 & Estudiante & $\begin{array}{c}\text { Trastorno del lenguaje } \\
\text { (UNE10) }\end{array}$ & $\mathrm{ADE}$ & $1^{\circ}$ & Hombre & 18 \\
\hline 15 & AE9 & Estudiante & Dislexia (UNE10) & $\mathrm{ADE}$ & $3^{\circ}$ & Mujer & 21 \\
\hline
\end{tabular}




\begin{tabular}{|c|c|c|c|c|c|c|c|}
\hline Id & Código & Rol & $\begin{array}{c}\text { Perfil y categoría } \\
\text { UNE EN 301549 } \\
(2008)\end{array}$ & Estudios & $\begin{array}{l}\text { Curso en } \\
2020-21\end{array}$ & Género & Edad \\
\hline 16 & AQ1 & Estudiante & $\begin{array}{c}\text { TEA y TDAH } \\
\text { (UNE10) }\end{array}$ & Química & $4^{\circ}$ & Mujer & 24 \\
\hline 17 & AD1 & Estudiante & $\begin{array}{c}\text { Dislexia y } \\
\text { disortografía (UNE10) }\end{array}$ & Derecho & $2^{\circ}$ & Mujer & 20 \\
\hline 18 & $\mathrm{AD} 2$ & Estudiante & $\begin{array}{l}\text { TOC, trastorno de la } \\
\text { alimentación, } \\
\text { depresión (UNE10) }\end{array}$ & Derecho & $1^{o}$ & Hombre & 19 \\
\hline 19 & AD3 & Estudiante & $\begin{array}{c}\text { Síndrome de Asperger } \\
\text { y TDAH (UNE10) }\end{array}$ & Derecho & $3^{\circ}$ & Hombre & 20 \\
\hline 20 & AD4 & Estudiante & Fibrosis quística & Derecho & $2^{\circ}$ & Mujer & 19 \\
\hline 21 & AD5 & Estudiante & $\begin{array}{l}\text { Baja visión, migrañas } \\
\text { y epilepsia (UNE02) }\end{array}$ & Derecho & $3^{\circ}$ & Hombre & 21 \\
\hline 22 & AD6 & Estudiante & $\begin{array}{l}\text { Lupus, Ictus y falta de } \\
\text { movilidad(UNE07 y } \\
08)\end{array}$ & Derecho & $4^{\circ}$ & Hombre & 42 \\
\hline 23 & AD7 & Estudiante & $\begin{array}{c}\text { Discapacidad auditiva } \\
\text { (UNE04) }\end{array}$ & Derecho & $3^{\circ}$ & Hombre & 48 \\
\hline 24 & AD8 & Estudiante & $\begin{array}{c}\text { Baja visión y parálisis } \\
\text { cerebral (UNE02, } 07 \text { y } \\
\text { 08) }\end{array}$ & Derecho & $1^{\mathrm{o}}$ & Hombre & 26 \\
\hline 25 & AD9 & Estudiante & $\begin{array}{l}\text { Trastorno límite de la } \\
\text { personalidad, dislexia } \\
\text { e hiperactividad } \\
\text { (UNE10) }\end{array}$ & Derecho & $1^{\circ}$ & Hombre & 42 \\
\hline 26 & AD10 & Estudiante & $\begin{array}{c}\text { Discapacidad auditiva } \\
\text { (UNE04) }\end{array}$ & Derecho & $4^{\circ}$ & Mujer & 27 \\
\hline 27 & AD11 & Estudiante & $\begin{array}{l}\text { Ansiedad, depresión, } \\
\text { anorexia nerviosa, } \\
\text { trastorno límite de la } \\
\text { personalidad y bulimia } \\
\text { (UNE10) }\end{array}$ & Derecho & $4^{\circ}$ & Mujer & 23 \\
\hline 28 & US1 & $\begin{array}{l}\text { Personal } \\
\text { de soporte }\end{array}$ & (1) & $\begin{array}{l}\text { Facultad de } \\
\text { Economía y } \\
\text { Empresa }\end{array}$ & - & Mujer & 73 \\
\hline 29 & US2 & $\begin{array}{l}\text { Personal } \\
\text { de soporte }\end{array}$ & - & $\begin{array}{l}\text { Facultad de } \\
\text { Derecho }\end{array}$ & - & Mujer & 53 \\
\hline 30 & US3 & $\begin{array}{l}\text { Personal } \\
\text { de soporte }\end{array}$ & - & $\begin{array}{l}\text { Facultad de } \\
\text { Química }\end{array}$ & - & Mujer & 62 \\
\hline 31 & US4 & $\begin{array}{l}\text { Personal } \\
\text { de soporte }\end{array}$ & - & SAE & - & Hombre & 41 \\
\hline 32 & US5 & $\begin{array}{l}\text { Personal } \\
\text { de soporte }\end{array}$ & - & $\begin{array}{c}\text { Facultad de } \\
\text { Geografía e } \\
\text { Historia / } \\
\text { Filosofía }\end{array}$ & - & Mujer & 54 \\
\hline 33 & US6 & $\begin{array}{l}\text { Personal } \\
\text { de soporte }\end{array}$ & - & & - & & \\
\hline 34 & US7 & $\begin{array}{l}\text { Personal } \\
\text { de soporte }\end{array}$ & - & $\begin{array}{c}\text { Facultad de } \\
\text { Economía y } \\
\text { Empresa / } \\
\text { Derecho / } \\
\text { Bellas Artes y } \\
\text { servicios } \\
\text { transversales }\end{array}$ & - & Mujer & 56 \\
\hline 35 & US8 & $\begin{array}{l}\text { Personal } \\
\text { de soporte }\end{array}$ & - & $\begin{array}{l}\text { Facultad de } \\
\text { Economía y } \\
\text { Empresa }\end{array}$ & - & Hombre & 51 \\
\hline
\end{tabular}




\begin{tabular}{|c|c|c|c|c|c|c|c|}
\hline Id & Código & Rol & $\begin{array}{c}\text { Perfil y categoría } \\
\text { UNE EN 301549 } \\
(\mathbf{2 0 0 8 )}\end{array}$ & Estudios & $\begin{array}{c}\text { Curso en } \\
2020-21\end{array}$ & Género & Edad \\
\hline 36 & PD1 & Docente & - & $\begin{array}{l}\text { Grado de } \\
\text { Derecho }\end{array}$ & - & Hombre & 67 \\
\hline 37 & PD2 & Docente & - & $\begin{array}{c}\text { Grados de } \\
\text { Investigación } \\
\text { privada, } \\
\text { Sociología y } \\
\text { Criminologia }\end{array}$ & - & Mujer & 58 \\
\hline 38 & PD3 & Docente & - & $\begin{array}{l}\text { Grado de } \\
\text { Derecho }\end{array}$ & - & Mujer & 27 \\
\hline 39 & PD4 & Docente & - & $\begin{array}{c}\text { Gradso de } \\
\text { Derecho, } \\
\text { Relaciones } \\
\text { laborales, } \\
\text { Ciencias } \\
\text { políticas y de } \\
\text { la } \\
\text { administración } \\
\text { y } \\
\text { Gestión de la } \\
\text { Administració } \\
\text { n pública }\end{array}$ & - & Mujer & 53 \\
\hline 40 & PD6 & Docente & - & $\begin{array}{l}\text { Grado de } \\
\text { Derecho }\end{array}$ & - & Mujer & 63 \\
\hline 41 & PD7 & Docente & - & $\begin{array}{l}\text { Grado de } \\
\text { Relaciones } \\
\text { laborales }\end{array}$ & - & Mujer & \\
\hline 42 & PD8 & Docente & - & $\begin{array}{l}\text { Grado de } \\
\text { Derecho }\end{array}$ & - & Hombre & 28 \\
\hline 43 & PD9 & Docente & - & $\begin{array}{l}\text { Grado de } \\
\text { Derecho }\end{array}$ & - & Hombre & 56 \\
\hline 44 & PE2 & Docente & - & $\begin{array}{l}\text { Grado de } \\
\text { Economía }\end{array}$ & - & Hombre & - \\
\hline 45 & PQ1 & Docente & - & $\begin{array}{l}\text { Grado de } \\
\text { Química }\end{array}$ & - & Mujer & 43 \\
\hline
\end{tabular}

Las entrevistas fueron realizadas por cinco de los investigadores. Para la conducción de las entrevistas se diseñó un formulario con una lista definida y ordenada de preguntas, sobre las cuales se incorporaron nuevas preguntas siempre que fue necesario para responder a las cuestiones relacionadas con el objeto de estudio. Las entrevistas se realizaron a través de la herramienta de videoconferencia Microsoft Teams o BlackBoard Collaborate y fueron grabadas y transcritas posteriormente por miembros del mismo equipo de investigación.

A partir de la transcripción se realizó un análisis temático y se buscó su asimilación con las buenas prácticas identificadas previamente (MASSAGUER et al., 2021). El análisis temático es un método cualitativo ampliamente utilizado (BOYATZIS, 1998, 4-5; 
ROULSTON, 2001, 280; BRAUN y CLARKE, 2006, 77; LEVITT et al., 2018, 28) para identificar y analizar temas y patrones en un conjunto de datos. En esta investigación se ha seguido el método propuesto por BRAUN y CLARKE (2006, 86-93) formado por las siguientes seis fases: 1) familiarización con los datos (transcripción, lectura relectura y anotación de ideas iniciales); 2) generación sistemática de códigos iniciales para las características más relevantes de los datos; 3) búsqueda de temas (recopilación de todos los datos relevantes para cada uno de los temas potenciales); 4) revisión de temas generando un mapa temático del análisis realizado; 5) definición y denominación de los temas; y 6) elaboración del informe (selección de extractos, análisis final de los extractos seleccionados, relación entre los resultados del análisis y las pregunta de investigación y la literatura publicada.

\section{Resultados}

Para cada una de las categorías identificadas en el estudio previo se mencionan aquellas buenas prácticas que han sido mencionadas por las personas implicadas en el curso de las entrevistas, y se incluyen algunas nuevas surgidas de sus propuestas. Para cada usuario que refuerza o valida una de las buenas prácticas se transcribe su aportación.

Tabla 2. Aspectos acerca de la planificación

Buenas prácticas

Estructura de la asignatura.

Explicar de manera explícita, con detalle y fácil de entender, la estructura de la asignatura.

\section{Comentario}

PE6: "Les enviaba correos a todos diciéndoles que la semana siguiente trataríamos tal tema, que sería interesante mirar una parte en casa".

PE6: "Les explicamos paso a paso [...] qué haríamos, qué temas entrarían, como sería el formato de la prueba, cuál sería la duración, como mínimo con dos semanas de antelación".

Pautas de organización EP.

Ofrecer pautas para la organización e informar de las prioridades.
PQ1: “En prácticas haciamos un seguimiento más personal. Con una estudiante de TDAH no la dejamos progresar hasta acabar las tareas anteriores... o sea cómo organizarle un poco la agenda”.

US3: “(A los estudiantes con TEA) les dije que lo mejor que podían hacer era organizarse un poco en forma de tablón, tareas pendientes, tareas iniciadas y tareas finalizadas". 


\section{Calendario.}

Ofrecer un calendario claro, incluyendo las fechas de realización de las clases en línea, de las entregas y las evaluaciones.

Calendario compartido (propuesta inexistente, que surgió a partir de los comentarios de US2).

Ofrecer un calendario compartido entre todas las asignaturas para que el estudiantado tenga una visión general de las pruebas a entregar cada semana.

Dar instrucciones de diferentes maneras (texto, audio o vídeo) sobre las tareas.

AA1: "Explicar los enunciados de las pruebas paso a paso”.

Formación al estudiantado sobre técnicas de organización.

Organizar formaciones sobre técnicas de organización para el estudiantado con diversidad funcional.

PE6: “Íbamos pautando mucho lo que tenía que ser cada semana de clase. Así creo que les ha ayudado mucho en la organización”.

US2: “Usamos una agenda electrónica en la que desde el primer día, antes de empezar las clases, los profesores deben indicar las fechas en las que el alumnado debe hacer alguna entrega, y asi está todo temporalizado”.

Fuente: elaboración propia

Tabla 3. Aspectos acerca del desarrollo de las clases en línea

\section{Buenas prácticas}

Disminución de la fatiga al reducirse el contacto social (propuesta inexistente, surgida

a partir de los comentarios de AA1).

No tener que relacionarse tantas horas y disminuir la presión que esto supone para rebajar la fatiga.

Canales de comunicación (propuesta inexistente, surgida a partir de los comentarios de PE3).

Creación de canales de comunicación entre estudiantes y profesorado durante las clases síncronas, en los momentos en que se están realizando prácticas para poder resolver dudas en el momento.

\section{Síntesis de la sesión.}

Compartir con el estudiantado un resumen o notas con los aspectos más importantes de la sesión. Por ejemplo, hacer resúmenes en audio de las sesiones de entre 10-15.

\section{Contenido de la sesión por escrito con previsión.}

Facilitar los materiales que se utilizarán o se expondrán (esquemas, presentaciones, enunciados de actividades...). De esta manera, pueden leerlo con anterioridad y con mayor tranquilidad sin la presión del directo.
AA1: “[en presencial] era agotador. [..] tengo que pensar cómo debo comportarme, cómo debo moverme, cómo debo hablar, que me están diciendo, que no me están diciendo...”.
PE3: "Tengo abierto el chat, y me podéis hacer preguntas. Me hacían. Pero no es lo mismo, claro”.
AA5: "Hacer resúmenes audio de las sesiones, cortos, de 10-15 minutos".
PD2: "Yo nunca había colgado documentos que redacto, y este año los he subido al campus".

PD3:" Siempre los colgaba al finalizar la clase. Ahora los he colgado con antelación”.

PD7: "Yo siempre cuelgo materiales que son de lectura simple [...] porque hay algunas lecciones que contienen mucha legislación [...] y les facilita mucho [...] tener un esquema" 
PE6: "Lo que ha ido muy bien es tenerlo todo disponible desde el inicio ...] para los alumnos NERE recomiendan que tengan todo el material con tiempo.

AA1: "Las paso en otro momento y las puedo volver

$$
\text { a ver". }
$$

AA4: "Las grabadas he tenido la facilidad, de poder repetir la grabación hasta entenderlo”.

Acceso a las sesiones grabadas.

Grabar y guardar el vídeo de las sesiones síncronas (o recurrir a grabaciones asíncronas),

adaptarlos y añadir subtítulos para

compartirlos con el estudiantado. El estudiantado podrá visualizar la sesión tantas veces como sea necesario.

AQ1: "Si me he distraído en algún momento, lo vuelvo a mirar".

PD4: "Pero esta persona, en este curso, puesto que grabamos las clases, tenía el audio que podía oír y por tanto no tuvo más complicaciones”.

US3: “Algunos profesores han grabado las sesiones con lo cual ellos [los estudiantes] han dicho que estaban muy contentos y que podian volver a repasarlas".

PE5: “[Grabe las sesiones] como mejora, las gravé siempre [...] porque todos me lo pedían [...] es una cosa que me gustaría ver instaurada".

Participación del intérprete de lengua de signos.

Incorporación de la interpretación en Lengua de signos en las sesiones docentes en streaming.
PD9: "Me tuve que adaptar, me lo pidió la intérprete, pues es que estar traduciendo agota mucho [.. ella me comentó que necesitaba un escanso [...] puse una actividad así los alumnos trabajaban y la intérprete descansaba".

Fuente: elaboración propia

Tabla 4. Aspectos acerca de las tutorías

Buenas prácticas

\section{Comentario}

AA1: "Estoy muy contento con los tutores NERE. Me han ayudado con profesores que no acaban de ver demasiado bien estas adaptaciones".

AE7: "Me ha sorprendido muchísimo porque he tenido dos profesores que me han enviado ellos

Contacto docente-estudiante a partir de tutorías en línea.

Mantener el contacto docente-estudiante (en ambas direcciones) a través de tutorías en

línea. El profesorado contacta al estudiante para entender sus necesidades.

Tutorías de inclusión (propuesta inexistente, surgida a partir de los comentarios de US3).

Incorporación del perfil de tutor/a de inclusión. mismos un correo y me han preguntado si necesitaba alguna cosa".

US3: "Según convenga, hacemos tutorías de refuerzo individuales o junto con el tutor académico, según si se trata de un problema académico y se debe de reducir el número de asignaturas, o si sólo es un problema de adaptación de aprendizajes y evaluaciones".

PE5: "Te permite bastante adaptarte al horario de la persona que te lo solicita si estás disponible”.

US3: “Ahora se incorporará en el PAT de la Facultad [...] la tutoría de inclusión”.

Fuente: elaboración propia 
Tabla 5. Aspectos acerca de la evaluación

Buenas prácticas

\section{Comentario}

PD4: "He realizado evaluación mixta con ejercicios de razonamiento y desarrollo y, en paralelo, exámenes tipo test [...] El examen tipo test era como el incentivo para que estudiaran y
Combinación de diversos instrumentos de evaluación en una misma asignatura (propuesta inexistente, surgida a partir de los comentarios de PD4 y PE1).

Combinación de exámenes tipo test y exámenes más reflexivos o de razonamiento en una misma asignatura. después el otro tenía como objetivo ver si habían entendido lo que habían estudiado y eran capaces de aplicarlo a un caso, en situaciones de razonamiento, más prácticas”.

PE1: "Lo único que he tenido que hacer en cuanto a adaptaciones es en el proceso de evaluación porque ha sido a través de tareas que eran cálculos, así como mediante una batería de test multirespuesta”.

AE5: "Este año tuve una prueba médica en la semana de exámenes presenciales y me la aplazó el

$$
\text { SAE”. }
$$

Flexibilidad en los exámenes por situaciones médicas (propuesta inexistente, surgida a partir de los comentarios de AE5 y PE1)

Ser flexibles en la programación de actividades en el caso de que algún estudiante no haya podido asistir a causa de una prueba o visita médica.

Adaptación al trabajo individual o en grupo.

Permitir trabajos en formato individual o en grupos reducidos (dos o tres personas como máximo) para el estudiantado que lo requiera.

Formación de equipos de trabajo según las características del alumnado (propuesta inexistente, surgida a partir de los comentarios de US3, PQ1 y AE4).

Propiciar un entorno favorable para el trabajo en grupo, ayudando a formar equipos de estudiantes según las características y necesidades de cada uno.
PE1: "Después he tenido otro alumno que no es NERE [...] y ha tenido que estar en cama (acabado de operar) [...] y ha pasado más de un mes y medio con este problema [...] cuando volvió en la semana

de exámenes tenía cinco pruebas, todas acumuladas y yo le dije, la mía, no la hagas [...] No puedes hacer cinco pruebas en dos horas [...] la mía ya la haremos más adelante".

PD4: "Por la dinámica de las actividades, no presencial sí que las hacemos [...] Digamos que ellos podían trabajar en grupo si querían, pero no era obligatorio. Algunas actividades las podían hacer en grupo, pero después las entregas debían ser individuales".

US3: "Los que tienen TDAH suponen un problema en el laboratorio porque son muy caóticos, más desordenados y entonces... pero como nosotros les dejamos trabajar en parejas con quien quieran a lo

largo de la carrera, con la ayuda de los compañeros supone un menor problema [...] y te diré, ni la dislexia, ni el TDAH está estigmatizado".

US3: " $Y$ entonces lo que hacemos es una asignatura que se llama gestión de proyectos [...], cuando tenemos la lista con todos los alumnos, hablo con los profesores que han tenido alumnos NERE para saber qué tipo de alumno es, y le buscamos un equipo en el que pueda encajar bien [...] intentamos buscar para ellos un entorno favorable".

PQ1: “(¿Problemas de interacción con sus grupos de trabajo?) No, porque hicimos un grupo de trabajo en paralelo y la estudiante tenía su propio grupo para trabajar, no estaba sola [...] es decir,

propuse una actividad de clase invertida en modalidad virtual, dentro de lo que se podía hacer, 
y para formar los grupos como y, como yo no les conocía, les pedía que se agruparan y ella estaba en uno de los grupos. No fue necesario que la colocase yo".

AE4: "Yo creo que la cosa es que se han hibernado los grupos, y hemos continuado con los que teníamos [...] Gracias a ello, porque presencialmente es diferente y a mi me cuesta adaptarme a la uni ya que hay mucha gente".

Evaluación oral o manuscrita.

Ofrecer la opción de evaluación oral o manuscrita (posteriormente digitalizada), cuando sea recomendable para determinados estudiantes.
AE1: "Los exámenes online me representan un esfuerzo inmenso porque no sólo hay un tiempo límite [...] porque con mis dificultades (sólo tengo una mano disponible) para escribir, escanear, enviar y todas esas cuestiones son más complicadas. No es lo mismo que un papel, lapiz y un boli [...] Eso sí, algunos profesores lo han entendido y han modificado el tipo de examen. Incluso me lo han enviado a mi correo electrónico, me lo he descargado, impreso y realizado a mano, escaneado y enviado en el tiempo pactado".

Aclaraciones sobre las preguntas de los exámenes.

Crear canales para que el estudiantado pueda solicitar aclaraciones de las preguntas durante el examen.
PQ1: "Para el parcial que fue online, lo que ofrecí a los alumnos y, a ella en particular, fue abrir una sesión en Collaborate por si tenían dudas. En su caso, le escribí un correo para informarle de que existía esta herramienta”.

Ampliación de tiempo.

Ampliar el tiempo para la realización de tareas académicas y exámenes escritos si el estudiantado lo necesita e indicar de manera clara el tiempo disponible para realizar la prueba.

Facilitación de lectura labial en pruebas en videoconferencias.

$\mathrm{Si}$ el estudiantado necesita realizar la prueba por videoconferencia, se debe facilitar la lectura labial y repetir la información tantas veces como sea necesario.

\section{Intérprete de lengua de signos.}

Ofrecer o permitir la presencia de un/a intérprete de lengua de signos durante el examen.

Tamaño de letra y contraste modificables.

En el documento de evaluación, el tamaño de la letra, el interlineado y su contraste se deben poder modificar.
PE1: "Hemos realizado adaptaciones consistentes en permitirle empezar antes el examen que al resto".

PD7: (sobre un estudiante con sordera) "Para él es importante que yo vocalice bien y que hable cerca de la pantalla. Por eso en las clases me pongo más cerca del monitor para que pueda leer bien los labios [...] desde la primera clase he intentado, sabiendo que tenía a este alumno, acercarme mucho para que pueda leer bien, intentar no ir demasiado rápido [...] y la verdad es que el me ha dicho que lo ha seguido bien".

PD9: "La intérprete estaba en el campus como cualquier otra alumna. Ella pidió acceso al campus y yo la incorporé. Ella podía entrar en el campus y ver el material [...] No tuve ningún problema”.

PD4: "Un estudiante que tenía estos problemas

visuales [...] necesitaba poder ver más (el entrevistado hace el gesto de ampliar con la mano) ... las pruebas se las teníamos que enviar en Word para que lo pudiera responder [...] en el mismo documento y ampliando el tamaño de la letra [...] también le puse un ejercicio específico para él". 
PD8: "Tuve que subir todos los archivos en dos versiones: PDF y Word por si ella quería cortar y pegar para situar el contenido en otro sitio".

Pruebas con la cámara encendida. cámara. Para las personas ciegas puede suponer una dificultad conseguir enfocar la cámara, y para algunas personas con trastornos mentales quizá prefieran no activarla.
Aplicar criterios de flexibilidad en el uso de la

US2: "Yo, por ejemplo, las reuniones personales, las tutorías, siempre las hago con el MS Teams, por si quieren, pueden preservar su intimidad bloqueando su espalda. Pienso que esto ayuda mucho ya que si una persona que ya tiene una serie de problemas encima tiene que avergonzarse de lo que tiene alrededor, ostras, complica mucho más la interacción”.

\section{Faltas de ortografía y expresión escrita.}

Tener en cuenta, en la evaluación de las actividades del estudiantado con dislexia, los errores y faltas de ortografía, así como la expresión escrita, que deberán ser excluidos del cálculo de penalizaciones aplicables en cada caso.
US3: “ Yo lo que pedía a los tutores, ya que también hacen una valoración, es que esta competencia no se valorara y que lo informaran a

los profesores ya que tengo dislexia y esto me conlleva dificultades para expresarme de la manera que se exige a otra persona que no tiene esta dificultad".

Fuente: elaboración propia

Tabla 6. Aspectos acerca de la metodología de enseñanza-aprendizaje

\section{Buenas prácticas}

Ejercicios adicionales optativos (propuesta inexistente, surgida a partir de los comentarios de PE2 y PE3).

Ofrecer ejercicios adicionales, optativos (con solucionarios), para que el estudiantado que lo requiera pueda realizarlos.

Evaluación continuada.

Priorizar la evaluación continua y rebajar el peso del examen, permitiendo adaptar mejor la evaluación a todo tipo de necesidades.

\section{Comentario}

PE2: "Tienen ejercicios adicionales con soluciones disponibles".

PE3: "No ha sido necesaria una adaptación específica porque ya tienen el libro, los powerpoints y además para este curso creamos material de autoestudio para todos los alumnos".

PD2: "He intentado evitar que hagan evaluación única. Siempre intento que hagan la continuada. Normalmente, no hago examen final y les hago trabajar mucho durante el curso".

Fuente: elaboración propia

Tabla 7. Aspectos acerca de los materiales accesibles

\section{Buenas prácticas}

Creación de materiales accesibles.

Crear documentos accesibles siguiendo las guías de creación de contenido accesibles y las buenas prácticas conocidas.

Vídeos de las partes prácticas de las asignaturas. (propuesta inexistente, surgida a partir de los comentarios de PE6).

Creación de vídeos de los temas más importantes de la asignatura, así como de

\section{Comentario}

AE4: "El problema del campus es, sobre todo, un problema de accesibilidad de los documentos.

Mientras que otro estudiante encuentra el artículo y ya está, yo, además de encontrarlo, tengo que poder leerlo".

PE6: "Hemos realizado vídeos [...]. Lo que hicimos los profesores de la asignatura fue crear vídeos expresamente sobre algunos temas, sobre todo, de los más potentes, los que tienen una parte práctica [...]. Los tenías disponibles en cualquier momento, los podían ver todas las veces que necesitases y creo que fue una ayuda muy importante que hemos 
Buenas prácticas

aquellos más prácticos. Que se encuentren disponibles en todo momento.

\section{Comentario}

mantenido hasta el día de hoy y queremos seguir manteniendo porque es un soporte que agradecen mucho. Si no fuera por la pandemia nunca nos habríamos planteado subir vídeos de nuestras clases. Quizá sea la adaptación más diferente”.
Documentos de texto no protegidos.

Crear los documentos de texto en formatos editables para que el estudiantado pueda cambiar su tamaño, interlineado, contraste.. Evitar bloquear o imponer medidas de seguridad.

Jerarquía y orden de los elementos.

En los documentos (enunciados de exámenes, presentaciones...) se deben cuidar aspectos como el título y la jerarquía de la diapositiva, la numeración, la descripción de las imágenes y la comprobación del orden de lectura de los elementos.
PE1: "Todos los documentos que hacemos, procuramos que no estuvieran protegidos. Mejor en formato Word o Powerpoint, que en PDF”.

PQ1: "En el examen final mi equipo docente decidió poner el formulario desordenado y yo lo puse en columnas pensando en ella [...] Yo imparto

Termodinámica. En esta asignatura tenemos mucha resolución de problemas y algunas fórmulas que damos a los estudiantes. En lugar de proporcionar enunciados y fórmulas juntos, les damos un formulario a parte para que puedan escoger la fórmula que deben utilizar. En cambio, algunos compañeros lo ponen todo junto. A ellos les va mejor tenerlo separado o, al menos, en dos columnas con un poco de separación para que lo puedan distinguir mejor".

Texto alternativo en las imágenes.

Añadir un texto alternativo breve y sintético (una frase o máxima dos) a todas las imágenes y contenido gráfico o acompañar las páginas con notas. Si la imagen contiene texto (frases o fórmulas matemáticas), debe incluirse en el texto alternativo.

Si se trata de un vídeo o de una imagen complejos (un gráfico, por ejemplo), debe redactarse un breve resumen como texto alternativo y complementarlo con una descripción larga en el cuerpo del documento.
PD8: “Soy una persona que trabaja mucho con imágenes y vídeos. Debía tener mucho cuidado los vídeos por si no eran suficientemente descriptivos.

En el modo en línea cosas como las reacciones o las interacciones sociales no se ven. En modalidad presencial me sentaba a su lado para hacerles una descripción del vídeo”.

AA5: "Suspendí Análisis de Datos porque las fórmulas, el formulario, los cuadrados, todas las

simbologías que utilizáis en estadística a mí el ordenador no me las lee, lo cual supone una odisea para mi".

Fuente: elaboración propia

Tabla 8. Aspectos acerca del acompañamiento emocional/social

\section{Buenas prácticas}

Atención y soporte al alumnado.

Atender las inquietudes y necesidades del estudiantado y estar abiertos a resolver sus dudas.

\section{Comentario}

PD2 "Haber sido coordinadora de tutores y tutora hace que la gente se dirija a mí".

PD3: "Lo que también estoy haciendo es enviar correos individuales a estos estudiantes cada mes y medio para preguntarles cómo les va la asignatura, ofrecerles tutorías... Podemos buscar fechas, que me pregunten [...] Unos días antes de los exámenes

enviaba un correo específico a estas personas informándoles que tenían un \% de tiempo adicional para sus exámenes de x minutos". 
Buenas prácticas

Encuentros grupales.

Encuentros grupales.

Crear un espacio virtual de encuentro grupal.

Grupo coordinado de profesorado (propuesta inexistente, surgida a partir de los comentarios de PD1).

Crear canales para que el profesorado pueda compartir y comentar casos concretos de estudiantes que comparten en diferentes asignaturas.

\section{Comentario}

PD7: "Yo creo que es lo que se tiene que hacer, estar pendiente del alumno para que en el momento que tu percibes un problema, darle algún tipo de solución [...] (sobre un estudiante con sordera) Yo lo vuelvo a repetir tantas veces como haga falta. Lo hago con cualquier alumno".

US3: "El grupo meetup es un grupo en el que nos reunimos una vez al mes y asistimos dos psicólogos de la Asociación Síndrome de Asperger de Cataluña, una persona del $S A E$, a veces viene $X X$, ahora viene $X X$ y yo como profesora UB. Les reunimos para que comenten sus problemas académicos [...] La mayoría están contentísimos".

PD1: "Los estudiantes de Derecho, en particular, tienen una característica diferente a todos los demás grupos [...] es que se trata de un grupo coordinado, es decir, todos los profesores cada semestre tenemos un chat y un coordinador específico para este grupo

[...] tenemos un contacto al que remitirnos para cuestiones técnicas [...] nos damos soporte mutuo".

Fuente: elaboración propia

Tabla 9. Aspectos acerca de la logística/aplicación de la adaptación

\section{Buenas prácticas}

Personal voluntario para dar soporte, transcribir o añadir subtítulos.

Organizar estudiantes (disponiendo de reconocimiento académico para tareas de voluntariado) o profesorado voluntario para

dar soporte, tomar apuntes o crear transcripciones o subtítulos durante una sesión.

\section{Ayudas con el equipamiento}

Convocar ayudas para el préstamo de equipamiento informático y soporte a la conectividad.

\section{Comentario}

AA3: "Tengo una compañera que me ayuda. Me dice lo que se cuelga, lo que se tiene que entregar.. Me ayuda a organizarme [...] Es una de las mejores de la promoción y me ayuda mucho [...] Del año pasado, por ejemplo, en Genética, tuve problemas para entender el temario y los ejercicios, pero mi compañera me ayudó".

US4: "También se destacaba el déficit de recursos técnicos de algunas personas para las cuales la UB implementó el programa Connecta UB en el que el SAE también colaboró".

Detectar estudiantes NERE por parte de la coordinación (propuesta inexistente, surgida a partir de los comentarios de PE5)

Tener detectado y localizado por parte de la coordinación al estudiantado NERE de todas

las asignaturas con el objetivo de poder realizar un mejor seguimiento.

Facilitar el acceso a software de las asignaturas (propuesta inexistente, surgida a partir de los comentarios de US4, US7 y US8).

Gestionar el acceso del estudiantado al software de la asignatura.

PE5: "Pero en el momento en el que nos encontramos con la pandemia, sí que hicimos un cambio de chip y me dije ¿qué pasará con estos alumnos?. Y fue cuando quise reunir a todos estos estudiantes [...] tenerlos a todos controlados".

US4: "Algunos incluso se han fijado en aquello que impulsó el CRAI en relación con el Blackboard y han dicho que les iba bien hasta el punto que lo comentaron al Centro de Recursos Educativos de la

ONCE y nos han pedido acceso para poder estudiarlo y, a partir de aquí, ver qué mejoras se pueden hacer e informar a sus alumnos futuros". 
US7: "Sí, porque en particular el alumno tenía problemas con un software específico que se utiliza en algunas asignaturas de la Facultad [...]. Por tanto, tuvimos que hacer gestiones con el proveedor, por un lado, para intentar resolver estos problemas $y$, por otro, con el alumno, para poder probar las soluciones [...]. Hicimos bastantes pruebas con el alumno y con su portátil y encontramos una solución que quizá le ha permitido trabajar un poco aunque no fuera una solución final".

US8: "En el grado en sociología tenemos cuatro asignaturas de técnicas de investigación que requieren el uso de un programa informático que se dedica al análisis estadístico y, claro, esto comporta una serie de clases presenciales prácticas con un ordenador que nos costó mucho adaptar desde casa".

Reducción de desplazamientos como manera de ganar tiempo (propuesta inexistente, surgida a partir de los comentarios de AA4 y PD2).

Disponer de más tiempo por el hecho de no tener que desplazarse.

\section{AA4: "Me ha beneficiado el disponer de más} tiempo. El tiempo de transporte...".

PD2: "Esto de la cámara es una buena opción para los NERE que tienen angustia por coger el tren... No está mal que se puedan conectar, pero se tendría que protocolarizar. Tenía un estudiante que venía de

Reus y que por una hora no le salía a cuenta. En este caso no me importa conectar la cámara, pero tendrían que ser casos muy concretos".

PD4: "Hay cosas que sí que las puedes adaptar (dislexias, discapacidades visuales...), pero otras, como dolencias más graves que... pienso que no tenemos formación y que requieren una persona de soporte mucho más directa [...] La verdad es que los cursos de ICE sobre estos temas técnicos los he hecho casi todos, y si te lo explican la verdad es que después es muy intuitivo y vas avanzando”.

Formación al profesorado (propuesta inexistente, surgida a partir de los comentarios de PD4, PD6 y US2).

Ofrecer pautas, tutoriales y formación al profesorado sobre aspectos relacionados con la accesibilidad.
PD6: "Fui a un curso sobre accesibilidad y, bueno, me vi incapaz de hacerlo tal y como decían que se tenía que hacer, pero bueno, ahora sé que és y que se puede pasar del escrito al oral y esto es altamente positivo".

US2: "No es tanto el campus, sino el desconocimiento del profesorado de las herramientas que ofrece. Algunas existen, pero nadie sabe que están allí. Por ejemplo, el tema de la ampliación de tiempo. Si lo supieran con antelación, no supondría tantos problemas."

Fuente: elaboración propia 
Tabla 10. Aspectos legales

Buenas prácticas

Comunicación sobre las adaptaciones.

Comunicar las adaptaciones al estudiantado con discreción y confidencialidad.

\section{Perfil afectado}

PE6: "Sí, en algunos casos sí que fue necesario hacer entradas en el campus virtual diferenciadas para los alumnos NERE, por ejemplo, para los que

podían tener más tiempo. En esos casos, les

proporcionábamos un acceso diferente con sus iniciales. El formato de la prueba era el mismo, pero tenían más tiempo”.

Fuente: elaboración propia

Tabla 11. Aspectos sobre los servicios de atención

Buenas prácticas

Adaptaciones necesarias.

Proporcionar soporte al profesorado en las adaptaciones necesarias de material.
Guía para el profesorado con pautas a seguir (propuesta inexistente, surgida a partir de los comentarios de PD8, PE2, PD4, US2 y US8).

Proporcionar al profesorado, antes del inicio de curso, un informe y una guía con pautas e instrucciones a seguir con el estudiantado con necesidades específicas que tendrán en el aula.no

\section{Perfil afectado}

PD1: "Sí, nos lo explicaron a principio de curso [...] a través del enlace a una macro".

PD6: "La chica que se encargaba [...] había sido alumna mía también y tiene mucho interés [...] además te dice que preguntes, que hagas...”.

PE3: "Los avisos que tenemos son cuando, desde el PAT, nos llegan los informes: tenéis tal alumno que tiene esta necesidad y su adaptación es esta. Esto está muy bien. Después, en ocasiones, se envía un mensaje al profesor o al tutor que tienen para recordarles, o presentarse [...] Yo lo agradezco realmente porque como tienes tantas cosas y tantos alumnos, a veces, se te olvida que tienes aquel alumno que ha precisado una adaptación y sí que se agradece cuando ellos te recuerdan, al acercarse un examen, que tienen aquella adaptación, porque están dentro del programa [...] porque es muy fácil olvidarte".

Servicios de atención accesible al estudiantado (propuesta inexistente, surgida a partir de los comentarios de AA4).

Disponer de un servicio de atención al que los estudiantes puedan acceder directamente. Por ejemplo, PIUNE de la UAB.

AA4: "La verdad es que desde el PIUNE me han gestionado cualquier petición que he hecho y lo han llevado super bien. No he tenido ningún problema".

PD8: "Otros años ha sido diferente, pero este año ha sido directamente la responsable del PAT NERE

la que nos envió un correo una semana antes de empezar con un resumen de las pautas que seguir”.

PE2: “Este alumno simplemente me envió un correo y yo, que tenía el informe del SAE, al pedirme un poco más de tiempo en el examen no tuve ningún inconveniente".

PD4: "Las instrucciones que recibimos cuando XXX nos informa que tenemos un estudiante NERE vienen acompañadas de un informe y, en este informe [...] se especifica cuáles son las particularidades y los problemas, las dolencias que tiene esa persona, qué hemos de tener en cuenta en... como impacta esto en la docencia, si hemos de tener en cuenta algún 
problema que se pueda producir en el desarrollo de la clase, o si tenemos que darle más tiempo en la realización de las pruebas de evaluación. Esto está bien porque te dice concretamente "debéis darle un $20 \%$ más que al resto”, lo calculas, se lo das y, en general, no hemos tenido problemas.

US2: "Yo lo hago de la siguiente manera [...] escribo un correo explicando que tendrán como alumnos a X y X, el SAE los ha valorado y les envío su informe, además le hemos preparado desde la Facultad, un plan personalizado de actuación. El plan personalizado es como un pequeño cuadro resumen [...] que se va actualizando, para que sepan [...] las cuatro pautas básicas".

US8: "Yo siempre me pongo en contacto con los profesores de este estudiante para decirles qué singularidad se encontrarán, paso el informe, etc. [...] le digo que recomendaciones existen, qué tipo de trastorno tiene, qué adaptaciones serían convenientes y, sobre todo, le animo y le digo que estoy a su disposición para resolver cualquier duda, que puede contactar con otros profesores que hayan tenido estudiantes con el mismo perfil por si requiere información más práctica. Esto especialmente en asignaturas más difíciles y para alumnos que presentan una especial dificultad [...] Antes de empezar cada semestre avisamos [...] a los profesores responsables de los grupos en qué tendrán docencia estos alumnos y antes de ello

Encuestas al estudiantado con diversidad funcional.

Pasar periódicamente una encuesta al estudiantado con diversidad funcional para detectar aquellas cosas que han funcionado y aquellas otras problemáticas.

Fuente: elaboración propia

US2: "Hace unos años diseñamos una encuesta anónima para pasar a los estudiantes NERE [...] Yo,

a partir de esta encuesta, paso un informe muy detallado de lo que ha pasado, lo que no ha pasado...".

Destaca en los resultados que, tanto el acompañamiento emocional/social, como los servicios de atención, son los que han sido más tratados en las entrevistas, en este último caso, surgiendo nuevas peticiones no recogidas en ninguno de los documentos analizados. Por el contrario, tanto la metodología de enseñanza-aprendizaje, como los aspectos legales, han sido escasamente mencionados durante las entrevistas. Tampoco la creación de materiales accesibles, originalmente con 20 recomendaciones, ha sido muy popular. La planificación, el desarrollo de las clases en línea y la evaluación sí que han recibido bastante atención, aunque diversas recomendaciones recogidas en las guías de buenas prácticas no han sido validadas por los y las interesadas. 


\section{Límites}

Este estudio es una primera exploración de las necesidades de las personas implicadas en la docencia en el caso de una docencia virtual de emergencia. Los límites de este estudio se concretan en la muestra, pequeña para poder hacer una generalización o para tener validez estadística cuantitativa. Ahora bien, gracias a la diversidad discursiva, y a la profundidad de las entrevistas a nivel cualitativo, el estudio ofrece una visión inicial de la experiencia de los y las implicadas rica y completa.

\section{Discusión}

En total hay un importante conjunto de cuestiones que tanto estudiantes, como docentes, como personal de gestión con diferentes responsabilidades han calificado como problemáticas durante el proceso de enseñanza-aprendizaje llevado a cabo a lo largo de estos meses de confinamiento.

Como primer aspecto a destacar hay que decir que el marco legal asimila el incumplimiento de adaptaciones en la evaluación con infracciones graves y, según los entrevistados, dicho incumplimiento ha sido una práctica común. También el marco legal recoge la obligatoriedad de que las tecnologías y espacios virtuales sean accesibles y los datos recogidos en las entrevistas no transmiten esta sensación, en absoluto. En este sentido y, como ya hemos visto en las competencias digitales incluidas en DigCompEdu e INTEF, no se hace suficiente énfasis en la formación en docencia inclusiva, cuestión que los propios implicados han sacado a relucir en las entrevistas.

Por lo que respecta al estudiantado con trastornos del aprendizaje (dislexia, TDAH), trastorno del espectro autista o enfermedades mentales en general, se ha destacado la necesidad de explicar, por parte del profesorado, de una manera más clara la estructura de la asignatura, el calendario previsto, las actividades e informarles de las prioridades en cada momento; todo ello con el objetivo de facilitar una visión global de la materia, así como la planificación y organización personal. Curiosamente, los perfiles de TEA (trastorno espectro autista) han valorado la tranquilidad del entorno hogar y la relajación de las obligaciones sociales como un aspecto positivo de la situación de la pandemia. 
Las clases en línea, a través de aplicaciones de videoconferencia, también han supuesto una barrera para una parte del alumnado, a pesar de estar explícitamente cubiertas en el marco legal. Entre los principales problemas detectados destacan: una calidad de vídeo, audio e iluminación insuficiente en diversos casos; o la no sincronización entre lo mostrado por pantalla y lo explicado. Las personas con discapacidades cognitivas expresan su dificultad para seguir las clases en directo y requieren con antelación los materiales que se utilizarán, así como una síntesis posterior de la sesión. Por su parte, las personas con discapacidades auditivas requieren subtitulación, poder leer los labios con garantías o la presencia de un intérprete de lengua de signos, requisitos, todos ellos, que no siempre se han podido cumplir. En el caso del alumnado con discapacidad visual, las principales barreras se han dado en el acceso a las herramientas de videoconferencia o a interferencias con sonidos del entorno.

Diversos estudiantes, especialmente aquellos con discapacidades cognitivas, así como personal con cargos de gestión, expusieron la importancia de las tutorías y de acercar profesorado y estudiantado con el objetivo de conocer mejor sus necesidades particulares.

Las pruebas de evaluación, otro de los aspectos enfatizados en el marco legal, han supuesto una importante barrera para todos los perfiles. En algunos casos, la prueba no se pensaba para ser accesible para todas las personas, es decir, no se exploraban formas alternativas de demostrar los conocimientos adquiridos, más allá de las tradicionales o estándares. Una aplicación rigurosa de la ley podría llegar a suponer la comisión reiterada de infracciones graves. La posibilidad de ampliar el tiempo disponible, contar con documentos con recomendaciones sobre cómo abordar la prueba, realizar simulaciones con anterioridad al examen o tener canales abiertos para plantear dudas durante éstos han sido cuestiones comunes entre diversas personas entrevistadas. Otros aspectos relacionados son la flexibilidad en el calendario ante la posibilidad de solaparse pruebas médicas importantes o la ampliación del tiempo de descanso entre pruebas de diferentes asignaturas.

Otra de las cuestiones más comentadas es la poca o nula accesibilidad de las aulas virtuales y los materiales docentes (propios y de terceros), de nuevo contraviniendo la exigencia de las disposiciones legales. Esta cuestión pone de manifiesto el escaso conocimiento que tiene el profesorado sobre la creación de materiales docentes accesibles 
que cubran las necesidades específicas de los diferentes perfiles de discapacidad. El problema no se encuentra tanto en la disponibilidad de recursos (existen múltiples guías para crear material accesible) sino, sobre todo, en el importante desconocimiento y la falta de formación en la materia (ROIG, RIBERA y MARTÍN, 2021) que, como hemos visto, tampoco está en vías de repararse con los modelos de formación digital existentes.

Finalmente, otro aspecto común en todas las entrevistas al estudiantado ha sido el pobre acompañamiento emocional/social con el que han contado en una situación de aislamiento como la vivida. Se han sumado el desconocimiento del profesorado, la poca empatía -en algunos casos- y un exceso de trabajo, unido todo ello a la exigencia de una adaptación inmediata a un nuevo escenario nunca antes planteado, que tampoco ha ayudado en este sentido.

\section{Conclusiones}

Tal y como ha sido puesto de manifiesto en el análisis del marco normativo y la legislación aplicable, las universidades tienen la obligación de garantizar la accesibilidad de sus entornos virtuales (sitios web, aulas docentes virtuales, espacios de trabajo...), sus clases (tanto presenciales como en remoto a través de aplicaciones de videoconferencia), los materiales docentes y de aprendizaje (propios o de terceros) y sus instalaciones. La situación vivida como resultado de la suspensión de la actividad presencial por la crisis sanitaria derivada del Covid-19 ha puesto a prueba, tanto a las universidades en general, como a sus servicios de atención al estudiantado con necesidades específicas, al personal de administración y servicios, a los cargos académicos y a los y las docentes. A través de esta investigación se validan las recomendaciones recogidas en diversas disposiciones y guías de buenas prácticas a partir de las opiniones de estudiantes, docentes y personal de soporte y se observa el peso relativo que las personas implicadas otorgan a las pautas a seguir. Es de destacar cómo el acompañamiento psicológico es una de las medidas más valoradas.

Las observaciones recogidas permiten detectar un incumplimiento grave del marco normativo existente y la necesidad de mejorar los planes de formación digital del profesorado para incluir la educación inclusiva. En este sentido, como principal línea de trabajo futuro, se trabajará en los centros y con diversos organismos relacionados con la 
inclusión en la educación para hacer llegar estas recomendaciones e intentar que se incorporen en las directivas, planes de formación y protocolos universitarios. Otra línea de trabajo futuro consiste en la colaboración con otras entidades para realizar más entrevistas en diferentes contextos y validar más extensamente los resultados obtenidos en esta primera exploración.

\section{Bibliografía}

AGUILERA-HERMIDA, A.P. (2020). College Students' use and acceptance of emergency online learning due to Covid-19, International journal of educational research open, 1 .

\section{https://doi.org/10.1016/j.ijedro.2020.100011}

BOYATZIS, R.E. (1998). Transforming qualitative information: thematic analysis and code development. Thousand Oaks, CA: Sage.

BRAUN, V. y CLARKE, V. (2006). Using thematic analysis in psychology. Qualitative research in psychology, 3(2), 77-101. https://doi.org/10.1191/1478088706qp063oa

CATALUNYA (2003). Decreto 246/2003, de 8 de octubre, por el que se aprueba el Estatuto de la Universidad de Barcelona. Diari oficial de la Generalitat de Catalunya. Núm. $\quad 3993, \quad 22$ octubre 2003. https://www.ub.edu/web/ub/ca/universitat/organitzacio/normatives/estatut/estatut.html

CATALUNYA (2014). LLEI 13/2014, del 30 d'octubre, d'accessibilitat. Diari oficial de la Generalitat de Catalunya. Núm. 6742, 4 novembre 2014, 1-39. http://portaldogc.gencat.cat/utilsEADOP/PDF/6742/1379017.pdf

COUNCIL OF EUROPE (2007). Resolution ResAP(2007)3 "Achieving full participation through Universal Design". https://search.coe.int/cm/Pages/result_details.aspx?ObjectID=09000016805d46ae

ESPAÑA (2001). Ley orgánica 6/2001, de 21 de diciembre, de universidades. Boletín oficial del estado, 24 diciembre 2001, 307, 49400-49425. https://www.boe.es/eli/es/lo/2001/12/21/6 
ESPAÑA (2008). Instrumento de ratificación de la Convención sobre los derechos de las personas con discapacidad, hecho en Nueva York el 13 de diciembre de 2006. Boletín $\begin{array}{llllll}\text { oficial del } & \text { Estado, } & 21 & \text { abril }\end{array}$ https://www.boe.es/boe/dias/2008/04/21/pdfs/A20648-20659.pdf

ESPAÑA (2010). Real Decreto 1791/2010, de 30 de diciembre, por el que se aprueba el Estatuto del Estudiante Universitario. Boletín oficial del estado, 31 diciembre 2010, 318. https://www.boe.es/eli/es/rd/2010/12/30/1791/con

ESPAÑA (2013). Real Decreto Legislativo 1/2013, de 29 de noviembre, por el que se aprueba el Texto Refundido de la Ley General de derechos de las personas con discapacidad y de su inclusión social. Boletín oficial del estado, 3 diciembre 2013, 289. https://www.boe.es/eli/es/rdlg/2013/11/29/1/con

ESPAÑA (2020). Real Decreto 463/2020, de 14 de marzo, por el que se declara el estado de alarma para la gestión de la situación de crisis sanitaria ocasionada por el COVID-19. BOE: boletín oficial del estado, 14 marzo 2020, 67. https://www.boe.es/eli/es/rd/2020/03/14/463/con

ESPAÑA (2020). Resolución de 2 de julio de 2020, de la Dirección General de Evaluación y Cooperación Territorial, por la que se publica el Acuerdo de la Conferencia Sectorial de Educación sobre el marco de referencia de la competencia digital docente. Boletín oficial del estado, 13 julio 2020, 191, 50638-50668. https://www.boe.es/eli/es/res/2020/07/02/(2)

ETSI (2018). EN 301 549: accessibility requirements for ICT products and services. v2.1.2. Brussels: European Telecommunications Standards Institute. https://www.etsi.org/deliver/etsi_en/301500_301599/301549/02.01.02_60/en_301549v0 20102p.pdf

HILL, K. y FITZGERALD, R. (2020). Student perspectives on the impact of COVID-19 on learning, AISHE-J: the all Ireland journal of teaching \& learning in higher education, 12(2), 1-9. Recuperado de: https://ojs.aishe.org/index.php/aishe-j/article/view/459 
INTEF (2012). Plan de cultura digital en la escuela. Madrid: Ministerio de Educación,

$\begin{array}{llll}\text { Cultura } & \text { y } & \text { Deporte. }\end{array}$

http://educalab.es/documents/10180/647889/PlanCulturaDigitalEscuelajulio+2014.pdf/3 dcdcc7d-aa21-4315-a89b-b23e97cff93e

INTEF (2012). Marco estratégico de desarrollo profesional docente.

INTEF (2017). Marco común de competencia digital docente. Madrid: Instituto Nacional de Tecnologías Educativas y Formación del Profesorado. Recuperado de: http://aprende.intef.es/sites/default/files/2018-05/2017_1020_Marco-Com\%C3\%BAnde-Competencia-Digital-Docente.pdf

LEVITT, H.M., BAMBERG, M., CRESWELL, J.W., FROST, D. M., JOSSELSON, R. Y SUÁREZ-OROZCO, C. (2018). Journal article reporting standards for qualitative primary, qualitative metaanalytic, and mixed methods research in psychology: the APA Publications and Communications Board task force report, American Psychologist, 73, 26-46. http://dx.doi.org/10.1037/amp000015

MASSAGUER, L., ALCARAZ MARTÍNEZ, R., RIBERA, M., SALSE, M. (2021). Docencia online para estudiantes con diversidad funcional en el contexto de la COVID19: medidas e indicaciones para el profesorado en las universidades españolas. Edutec (en revisión).

PUNIE, Y. y REDECKER, C. (eds.) (2017). European framework for the digital competence of educators: DigCompEdu. Luxembourg: Publications Office of the European Union. http://dx.doi.org/10.2760/178382

ROIG, J., RIBERA, M. Y MARTÍN, D. 2021. Analysis on the creation of accessible documents with Microsoft Word online in the field of education. En: Proceedings of the XXI International Conference on Human Computer Interaction (Interacción '21). Association for Computing Machinery, New York, NY, USA, Article 1, 1-4. https://doi.org/10.1145/3471391.3471423 
ROULSTON, K. (2001). Data analysis and 'theorizing as ideology', Qualitative research, 1(3), 279-302. https://doi.org/10.1177/146879410100100302 